\title{
Unidirectional Side Scattering of Light by a Single-Element Nanoantenna
}

\author{
Dries Vercruysse, ${ }^{*}+, \dot{\dagger}$ Yannick Sonnefraud, ${ }^{\S}$ Niels Verellen, ${ }^{*}, \dagger \dagger$ Fabian B. Fuchs, ${ }^{\S}$ Giuliana Di Martino, ${ }^{\S}$ \\ Liesbet Lagae, ${ }^{\dagger, \ddagger}$ Victor V. Moshchalkov, ${ }^{\ddagger}$ Stefan A. Maier, ${ }^{\S}$ and Pol Van Dorpe ${ }^{\dagger, \ddagger}$ \\ ${ }^{\dagger}$ IMEC, Kapeldreef 75, B-3001 Leuven, Belgium \\ ${ }^{\ddagger}$ INPAC and Department of Physics, KU Leuven, Celestijnenlaan 200 D, B-3001 Leuven, Belgium \\ ${ }^{\S}$ Experimental Solid State Group, Physics Department, Imperial College London, London SW7 2AZ, United Kingdom
}

\section{Supporting Information}

\begin{abstract}
Unidirectional side scattering of light by a singleelement plasmonic nanoantenna is demonstrated using full-field simulations and back focal plane measurements. We show that the phase and amplitude matching that occurs at the Fano interference between two localized surface plasmon modes in a V-shaped nanoparticle lies at the origin of this effect. A detailed analysis of the $\mathrm{V}$-antenna modeled as a system of two coherent point-dipole sources elucidates the mechanisms that give rise to a tunable experimental directivity as large as $15 \mathrm{~dB}$. The understanding of Fano-based

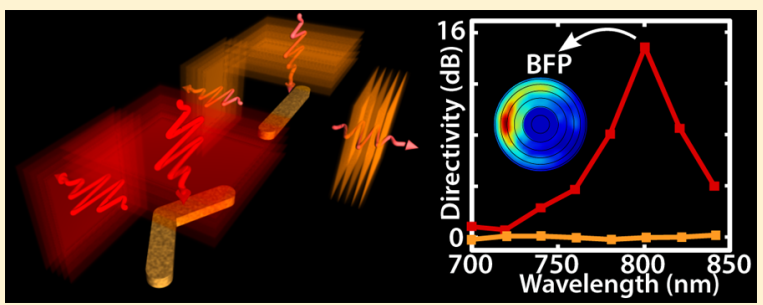
directional scattering opens a way to develop new directional optical antennas for subwavelength color routing and selfreferenced directional sensing. In addition, the directionality of these nanoantennas can increase the detection efficiency of fluorescence and surface enhanced Raman scattering.
\end{abstract}

KEYWORDS: Nanoantenna, surface plasmon resonance, directionality, Fano resonance, side scattering

$\mathrm{T}^{\mathrm{s}}$ he interaction of light with metal nanoparticles is largely governed by resonant oscillations of the free electrons at the metal-dielectric interface. These so-called localized surface plasmon resonances (LSPR) can reach frequencies in the visible spectrum, have large extinction cross sections, are very sensitive to the surrounding medium, and lead to deepsubwavelength electromagnetic field confinement and enhancement. Plasmonic resonators, therefore, bring optics into the nanoscale and have already found applications in disease diagnostics and treatment, photovoltaics, and optical communications. $^{1-7}$

One of the most determinative characteristics of a plasmonic resonator is its shape. It is well-known that the shape determines-to a large extent-the LSPR spectral positions. ${ }^{8}$ Specific resonator designs, consisting of a single or multiple particles, also allow to control the LSPR quality-factor by scattering loss engineering based on plasmon hybridization, ${ }^{9}$ sub- and superradiance, and Fano interference. ${ }^{10-13}$ Additionally, similar to classical antennas, a proper plasmonic antenna design will impact its directionality - that is, the ability to direct scattered radiation in a particular direction. Achieving high directivities in combination with a high degree of flexibility for the direction is elementary to devise efficient subwavelength plasmonic transmitters, receivers, and sensors.

To obtain directional scattering, constructive and destructive interferences of multiple coherent radiation sources with carefully designed spatial separation and phase differences are required. Directional scattering of a plane wave along its propagation direction has recently been observed in core-shell nanoparticles, ${ }^{14}$ as well as in nonmetallic silicon nanospheres. ${ }^{15}$ The obtained large forward-to-backward scattering ratios were shown to result from interfering dipoles and quadrupoles where retardation of the incident light over the particle volume activates the higher order mode and induces the required phase differences. ${ }^{16}$ Higher order modes in a tilted plasmonic nanocup can also rotate the scattering distribution away from the incident light direction. ${ }^{17-19}$ To favor unidirectionality perpendicular to the incident plane wave direction - that is, side scattering-the antenna's mirror symmetry has to be broken relative to the light polarization. This is possible for particle assemblies. A well-known example is the plasmonic Yagi-Uda antenna. $^{20-23}$ A more compact alternative consisting of a bimetallic nanodisk dimer was recently shown to route different colors either left or right from the incident light direction as a result of material-induced phase shifts between the two disks' dipole modes. ${ }^{24,25}$ Directional scattering under localized excitation of a plasmonic resonator, using, for example, fluorescent molecules or quantum dots, relaxes the structural requirements as the retardation of the excitation field over the particle volume introduces additional phase shifts and dipoleactivation of dark modes. ${ }^{20,26-29}$

Received: May 22, 2013

Revised: July 12, 2013

Published: July 25, 2013 
In this Letter, we demonstrate strongly unidirectional side scattering of a plane wave by an individual V-shaped metal nanoparticle, as illustrated in Figure 1a. This simple planar
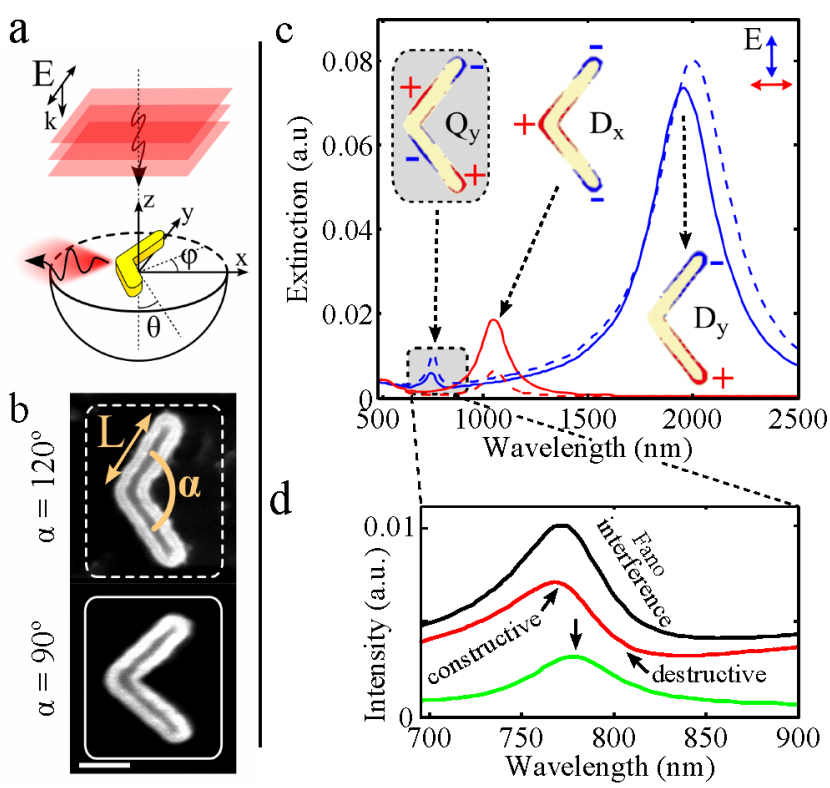

Figure 1. (a) Unidirectional side scattering of a plane wave with a plasmonic V-antenna. (b) SEM images of V-antennas with arm length $L=250 \mathrm{~nm}$ and opening angle $\alpha=120^{\circ}$ and $90^{\circ}$. Scale bar: $100 \mathrm{~nm}$. (c) Simulated extinction spectra for the antennas in panel $\mathrm{b}$ (dashed curves: $\alpha=120^{\circ}$, solid curves: $\alpha=90^{\circ}$ ) for $x$ - and $y$-polarization (red and blue, respectively). The insets show the charge density distributions of the observed V-antenna LSPR modes: $\mathrm{Q}_{y}, \mathrm{D}_{x}$, and $\mathrm{D}_{y}$. (d) A zoom-in on the $\mathrm{Q}_{y}$ mode $\left(\alpha=120^{\circ}\right)$ in panel c reveals Fano interference when comparing the extinction (black), scattering (red), and absorption (green) intensity.

single-element antenna geometry has been used before as a tunable-phase antenna building block in birefringent metasurfaces and aberration-free ultrathin flat lenses at telecom frequencies. ${ }^{30,31}$ While these applications rely on diffractive farfield interferences generated by carefully designed arrays, here, the broken mirror symmetry of a single $\mathrm{V}$-antenna allows us to benefit directly from the characteristic phase shifts of an intrinsic Fano interference, arising from the coherent near-field coupling of a dipole and higher order LSPR mode-even at near-infrared and visible frequencies. We use finite difference time domain (FDTD) calculations to analyze the antenna modes and map the three-dimensional far-field scattering intensity distributions. These results are then compared with, and confirmed by, experimental extinction spectroscopy and angular resolved scattering distributions obtained with back focal plane (BFP) imaging. ${ }^{20,24,32}$ The tunability of the Vantenna's directivity and scattering efficiency is further investigated using different V-shapes. Although being intimately related to the interference of coupled dipole and quadrupolar mode components, we will illustrate how a simple, intuitive two point-dipole model is capable of conveying the main aspects of the $\mathrm{V}$-antenna's directional behavior.

Scanning electron beam microscopy (SEM) images of two Vantennas studied in this work are shown in Figure $1 \mathrm{~b}$. The arm length, $L$, and opening angle ( $\mathrm{V}$-angle) of the arms, $\alpha$, define the antenna geometry. The nanostructures consist of sputtered gold with a thickness of $50 \mathrm{~nm}$ (= antenna thickness) and are supported by a glass substrate coated with $10 \mathrm{~nm}$ indium tin oxide (ITO) which facilitates SEM analysis. They were fabricated using electron beam lithography with negative-tone hydrogen silsesquioxane (HSQ) resist, subsequent $\mathrm{Xe}$ ion milling, and a sulfur hexafluoride + oxygen dry etch (Oxford instruments Plasmalab System 100 ICP 180). ${ }^{33}$ This last step is applied to remove the remaining resist on top of the particles. The antennas are arranged in $50 \times 50 \mu \mathrm{m}^{2}$ square arrays with a pitch of either $1.5 \mu \mathrm{m}$ for extinction spectroscopy or $4.5 \mu \mathrm{m}$ for BFP measurements.

Extinction (1 - Transmission) spectra are taken with a Fourier transform infrared (FTIR) microscope (Bruker vertex $80 \mathrm{v}+$ Hyperion) using two $15 \times$ magnification Cassegrain condensers. The scattering directions of the antennas are measured using back focal plane (BFP) imaging. ${ }^{20,32}$ Monochromatic light, selected from a Fianium supercontinuum laser source using a prism system, is delivered to the BFP setup with an optical fiber and focused on the sample by a low NA objective, after passing through a polarizer. The resulting incident beam has a narrow angular distribution (i.e., nearly parallel beam) and creates an illumination spot of $\pm 10 \mu \mathrm{m}$ diameter on the sample. The light scattered and directly transmitted into the glass substrate is collected with a $100 \times$ magnification oil immersion objective with an NA of 1.49. The $\mathrm{BFP}$, which contains the angular scatter information, forms inside the objective and is projected on an opaque disk that blocks the bright central spot corresponding to the unscattered light. This filtered image is then projected on a CCD camera. For more details and a schematic drawing of the setup, see Supporting Information.

A commercial FDTD solver ${ }^{34}$ is used to calculate extinction, scattering, and absorption spectra, LSPR charge density distributions, and far-field scattering distributions. The latter are either obtained with the built-in far field projection calculator of the solver for antennas in a homogeneous medium (i.e., no substrate) or calculated from the electric field intensities on a $3 \mu \mathrm{m}$ box surrounding the particle when the substrate is included (see Supporting Information for more details). The charge plots are obtained from evaluating the Poisson equation using the complex fields extracted from the simulations. The mesh size used in the simulations is $2 \mathrm{~nm}$. The dielectric permittivity of gold is based on Johnson and Christy data, ${ }^{35}$ and the refractive index of the substrate is $n=1.52$. The $10 \mathrm{~nm}$ ITO layer was not included in the simulations.

The scattering behavior of a nanoantenna is determined by its LSPR modes. For the V-antennas in Figure $1 \mathrm{~b}$, three strong resonant modes are observed in the simulated extinction spectra (Figure 1c). Corresponding modal charge distributions in the insets reveal these as: a dipole mode, $\mathrm{D}_{x}$, for $x$-polarized illumination (red curves), and for $y$-polarization (blue curves) the fundamental dipole mode, $\mathrm{D}_{y}$, and first higher order mode, referred to as $\mathrm{Q}$. The latter can be considered as the third order $(l, m)=(3,0)$ nanorod LSPR mode with an additional quadrupole component $(l, m)=(2, \pm 1)$ introduced by the structural symmetry reduction. Here, $l$ and $m$ refer to the spherical harmonics $Y_{l}^{m}$ in the Mie expansion of the modes. ${ }^{36}$

Spectral tunability of these antenna modes is most effectively achieved by varying the antenna length $L$ and is similar as for the well-documented nanorod antennas (Figure S2a in the Supporting Information shows how the modes red-shift with increasing $L) .{ }^{37}$ As the spectra in Figure 1c show, however, changing the $\mathrm{V}$-angle $\alpha$ from $120^{\circ}$ (dashed curves) to $90^{\circ}$ (solid curves) has little effect on the LSPR wavelengths (see also Figure S2b in the Supporting Information for different $\alpha$ ). 



Figure 2. Scattering intensity distributions and directivity of a V-antenna on a substrate $\left(L=250 \mathrm{~nm}, \alpha=120^{\circ}\right)$. (a) Simulated 3D distributions. Top: directional scattering at $\lambda=804 \mathrm{~nm}$. Bottom: no directionality at $\lambda=743 \mathrm{~nm}$. The light blue plane indicates the substrate interface. (b) Simulated 2D projections at several wavelengths throughout the $Q_{y}$-Fano resonance-indicated with the colored dots in the antenna's extinction spectrum (black curve). Insets show the cross-section of the 3D distribution at $\varphi=0(x-z$ plane $)$-dotted line: substrate/air interface. Directivity, $D$, calculated from the $2 \mathrm{D}$ polar plots is shown together with the extinction spectrum (green curve, right axis). (c) Corresponding experimental back focal plane (BFP) measurements. Color scale indicates normalized intensity. All data presented is based on $y$-polarized light.

Changing $\alpha$ does, nevertheless, considerably influence the extinction intensities and quality factors (Q-factors). As can be seen in Figure 1c, when $\alpha$ decreases the extinction intensity of $\mathrm{D}_{x}$ increases, while decreasing for $\mathrm{D}_{y}$ and $\mathrm{Q}_{y}$. This $\alpha$ dependence is a direct consequence of the changing effective dipole moments of the modes and their projection on the excitation field. ${ }^{38}$ Here, a larger dipole moment leads to a stronger LSPR excitation and hence a larger extinction crosssection. In addition, the variable dipole moments also affect the radiative losses and therefore the $\mathrm{Q}$-factor of the modes. The LSPR Q-factors as a function of $L$ and $\alpha$ are shown in the Supporting Information, Figure S2c. We conclude that, although the $\mathrm{V}$-angle $\alpha$ provides nearly no tunability of the LSPR spectral positions, it does affect the relative expression of the interfering $y$-polarized modes, and therefore, as will be discussed in Figure 3, the directionality of the antenna.

As already mentioned in the introduction, unidirectional side scattering requires an arrangement of multiple coherent radiation sources which, additionally, has broken mirror symmetry across the (E,k)-plane (Figure la). In the V-antenna this task can be fulfilled by the spectrally overlapping $\mathrm{D}_{y}$ mode and quadrupole $(l, m)=(2, \pm 1)$ component of the $Q_{y}$ mode. As the spectral zoom-in in Figure 1d shows $\left(\alpha=120^{\circ}\right)$, the coherent excitation of these modes results in interference in the scattering tail (red line) of the $\mathrm{D}_{y}$ mode. On the shorter wavelength side of the $\mathrm{Q}_{y}$ absorption resonance (green curve) constructive interference leads to increased scattering intensity, while on the longer wavelength side both modes interfere destructively. This phenomenon, characterized by an asymmetric spectral line shape, is known as Fano resonance or Fano interference and mainly arises here from the $\mathrm{Q}_{\text {y }}$ mode's $l=3$ component, as it is also observed for nanorods $\left(\alpha=180^{\circ}\right) .^{39}$
The impact of this Fano interference on the angular distribution of the scattering intensity is illustrated in Figure 2. In this figure, simulated (panels $a, b$ ) and experimental (panel c) results for the $L=250 \mathrm{~nm}, \alpha=120^{\circ}$ antenna in Figure 1 are provided, at several wavelengths around the $\mathrm{Q}_{\mathrm{y}}$-Fano resonance for $y$-polarized light. The strongest directionality in the simulations is reached at $\lambda=804 \mathrm{~nm}$. The corresponding three-dimensional (3D) far-field scattering intensity distribution in panel a (with azimuthal angle $\varphi$ and polar angle $\theta$ ) allows us to evaluate the scattering in every direction. Strong lateral directionality is clearly observed. Since, in general, plasmonic nanoparticles scatter most of the light into the higher index substrate, indicated by the light blue plane, the ideal scattering pattern expected for a particle in free space is disturbed. If no substrate were present here, the maximum scattering direction would shift up to $(\varphi, \theta)=(\pi,(\pi / 2))$, i.e., in the direction of the tip of the $\mathrm{V}$-antenna (negative $x$-direction). This means that we obtain directionality which is perfectly perpendicular to the incident light. A scattering plot near a quadrupolar resonance typically comprises four lobes. For the $\mathrm{Q}_{y}$ mode of the $\mathrm{V}$-antenna, this is most clearly seen in the $3 \mathrm{D}$ scattering plot at $\lambda=743 \mathrm{~nm}$ (Figure 2a, bottom): two lobes in $x$-, and two in $y$-direction. However, radiation in the $y$-direction $(\varphi= \pm(\pi / 2))$ is much weaker and not unidirectional because the antenna geometry, and hence the mode, has $y=0$ as symmetry plane.

To facilitate comparison with the experimental BFP images, the $3 \mathrm{D}$-plots are transformed into $2 \mathrm{D}$ projections, as shown in Figure 2a. The color scale indicates the normalized scattering intensity. The finite NA $=1.49$ of the collection objective and the refractive index of the glass substrate $(n=1.52)$ were taken into account in the calculation of these projections by including only scattering up to an angle $\theta=a \sin (1.49 / 1.52)=78^{\circ}$. 
Simulated BFP projections at different wavelengths are shown in Figure $2 \mathrm{~b}$. The colored dots indicate their spectral position in the corresponding extinction spectrum. The insets show the scattering intensity in a cross-section at $\varphi=0(x-z$ plane $)$ taken from the $3 \mathrm{D}$ distribution. The dotted line indicates the substrate/air interface.

To quantify the directionality of the antenna, we introduce the directivity, $D$, as the ratio of the light intensity scattered in the negative $x$-direction to the intensity scattered in the positive direction:

$$
D=10 \log _{10} \frac{\iint_{\left(\theta_{\mathrm{m}}-\delta, \pi-\delta\right)}^{\left(\theta_{\mathrm{m}}+\delta, \pi+\delta\right)} S(\varphi, \theta) \sin (\theta) \mathrm{d} \varphi \mathrm{d} \theta}{\iint_{\left(\theta_{\mathrm{m}}-\delta,-\delta\right)}^{\left(\theta_{\mathrm{m}}+\delta,+\delta\right)} S(\varphi, \theta) \sin (\theta) \mathrm{d} \varphi \mathrm{d} \theta}
$$

with $S(\varphi, \theta)$ as the intensity in the BFP image. The considered integration intervals are indicated in Figure $2 \mathrm{~b}$ (brown dot). $\delta=$ $10^{\circ}$, and $(\varphi, \theta)=\left(\pi, \theta_{\mathrm{m}}\right)$ corresponds to the maximum scattering intensity direction which is close to $\theta_{\mathrm{m}}=55^{\circ}$ for the simulations and $\theta_{\mathrm{m}}=45^{\circ}$ for the measurements. Alternative directivity calculations are provided in the Supporting Information. Directivity values derived from the polar plots in panel $b$ are shown together with the antenna's extinction spectrum (green line, right axis).

Far away from the $Q_{y}$ extinction resonance (brown and blue dots) very little directionality is observed. A nearly equal amount of light is scattered in the positive and negative $x$ direction. When approaching the LSPR resonance, gradually more power is scattered into the negative $x$-direction. The directivity reaches a maximum of more than $20 \mathrm{~dB}$ at $\lambda=804$ $\mathrm{nm}$, on the longer wavelength flank of the $\mathrm{Q}_{y}$ absorption resonance peak.

An excellent qualitative agreement with the experimental BFP images, and derived directivities, in Figure $2 \mathrm{c}$ is observed. Again, the colored triangles indicate in the experimental extinction spectrum the wavelengths at which the images where taken. Clear unidirectional side scattering is seen from 760 to $820 \mathrm{~nm}$, while wavelengths on the blue side of the $Q_{y}$ mode show an increasing amount of scattering in the positive $x$ direction. An experimental directivity of $15 \mathrm{~dB}$ is reached at $\lambda=$ $800 \mathrm{~nm}$. This value is comparable to directivities observed for bimetallic nanodisk dimers. ${ }^{24}$ Since the antennas are fabricated in a square grid with a $4.5 \mu \mathrm{m}$ pitch, the raw images are the combination of a square diffraction pattern with the scattering pattern of an individual antenna. Therefore, for clarity, values in between the diffraction spots were interpolated (see Supporting Information for more details).

The influence of a varying opening angle $\alpha$ on the total scattering cross-section of the $\mathrm{V}$-antenna was already discussed in Figure 1c. Spectral shifts of the $Q_{y}$ mode were found to be very small. Nevertheless, the distribution of the oscillating charges (approximate arrangement of the coherent radiation sources: $\mathrm{Q}_{y}$ and $\mathrm{D}_{y}$ ) changes, and consequent changes in the directionality are expected. Indeed, as Figure 3a shows, scattering distributions of antennas with decreasing $\alpha$, taken at their most directive wavelength, present important differences. For $\alpha=180^{\circ}$ the antenna is symmetric, and an equal amount of light is scattered left and right. In addition to the central scattering lobe $(\varphi=0, \pi)$, side lobes expected for the $l=$ 3 nanorod LSPR mode near $\varphi= \pm(\pi / 2)$ are observed. ${ }^{40}$ Once the antenna symmetry is broken $\left(\alpha<180^{\circ}\right)$, the right scattering lobe disappears. Figure $3 \mathrm{~b}$ shows the spectral behavior of the directivity for the antennas in panel a. It is seen that each

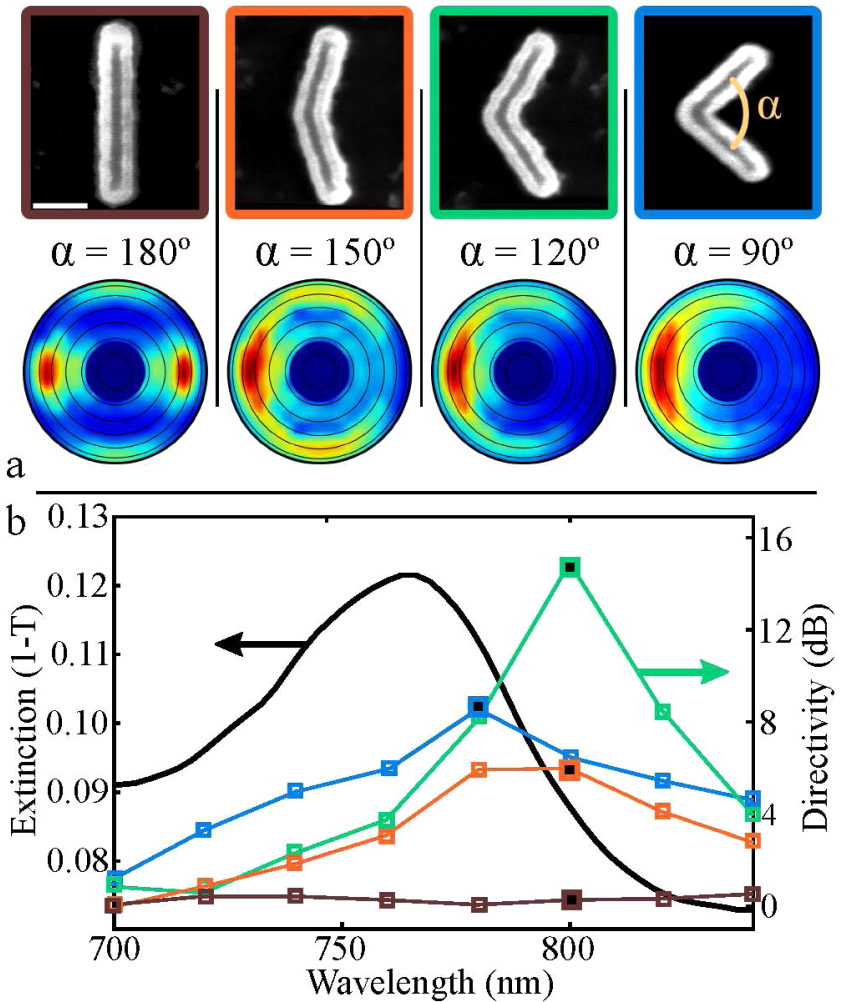

Figure 3. Directivity tunability with $\mathrm{V}$-antennas of varying opening angle $\alpha(L=250 \mathrm{~nm})$. (a) Back focal plane (BFP) images of the antennas shown in the SEM images at their most directive wavelengths, indicated in panel b. Scale bar: $100 \mathrm{~nm}$. (b) Spectral behavior of the directivity for $\alpha=180^{\circ}$ (brown), $150^{\circ}$ (orange), $120^{\circ}$ (green), and $90^{\circ}$ (blue). The black curve is the experimental extinction spectrum for $\alpha=120^{\circ}$.

asymmetric antenna can scatter directionally and reaches a maximum directivity at the longer wavelength flank of the $Q_{y}$ extinction resonance (black curve). The highest directivity of more than $15 \mathrm{~dB}$ is reached for $\alpha=120^{\circ}$. Further optimization of the antenna shape could push this value to even higher $\mathrm{dB}$. As, for decreasing $\alpha$, the antenna arms align with the $x$-axis, their individual $x$-oriented dipole moments, $p_{x}$, increase. This allows the antenna to radiate more power in the side lobes. Consequently, we see that for $\alpha=90^{\circ}$ scattering in the $y$ direction becomes again more prominent (panel a). As a result of the decreasing $\mathrm{Q}_{y}$ extinction cross-section for decreasing $\mathrm{V}$ angles (Figure 1c), antennas with $\alpha<90^{\circ}$ were not measured.

The observed directionality of the $\mathrm{V}$-antenna arises from the interference of two coherent radiation sources: $\mathrm{D}_{y}$ and $\mathrm{Q}_{y}$. It is in fact possible to substitute this coupled dipole-quadrupole system with two coherent dipole sources. The advantage of this representation is that it allows us to understand and describe the antenna's directional behavior with an intuitive two-dipole model (Figure 4a). ${ }^{24}$ The emission from two coherent point dipoles, separated by a distance $d$, is the sum of their individual waves. For two dipoles of equal strength, the power radiated in the two opposite directions along the axis that connects them, is proportional to $\cos [(\Delta \phi+k d) / 2]$ in one direction, while proportional to $\cos [(\Delta \phi-k d) / 2]$ in the other. Here, $\Delta \phi$ is the phase difference between the dipoles and the wavenumber $k$ $=2 \pi / \lambda$. The phase difference, $k d$, as a result of the spatial separation adds to $\Delta \phi$ and when either $(\Delta \phi+k d)$ or $(\Delta \phi-$ $k d$ ) becomes equal to $\pi$, the sources interfere destructively in 
$\mathrm{a}$
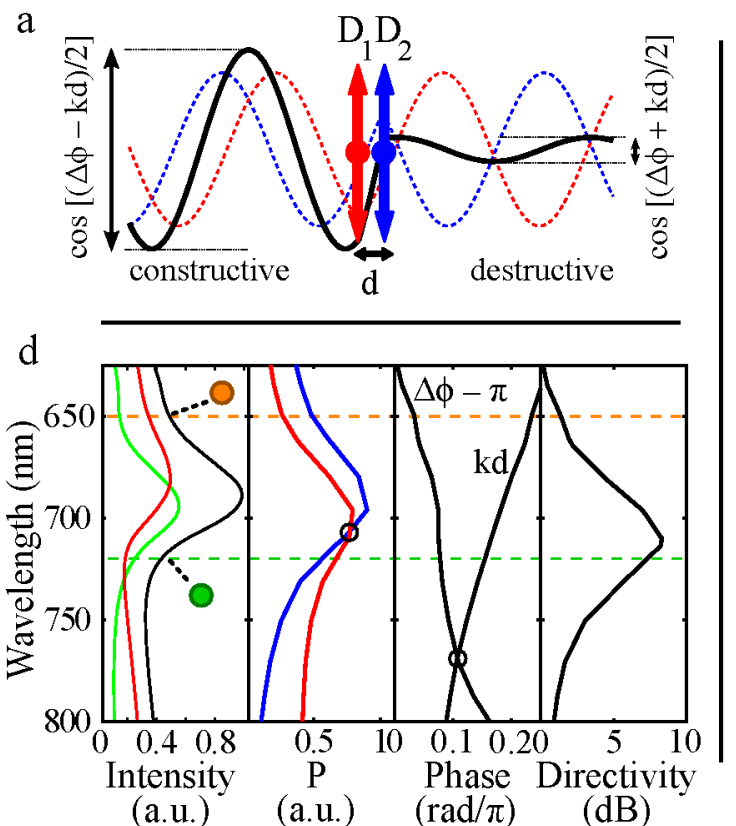

b

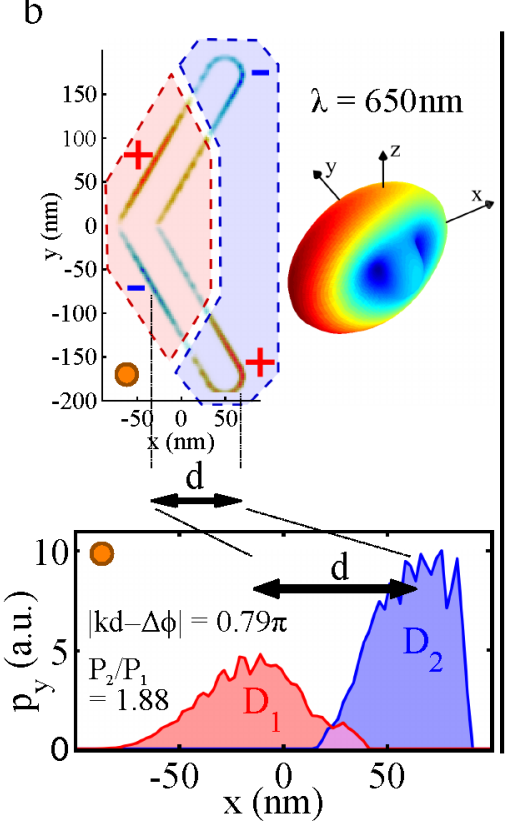

c

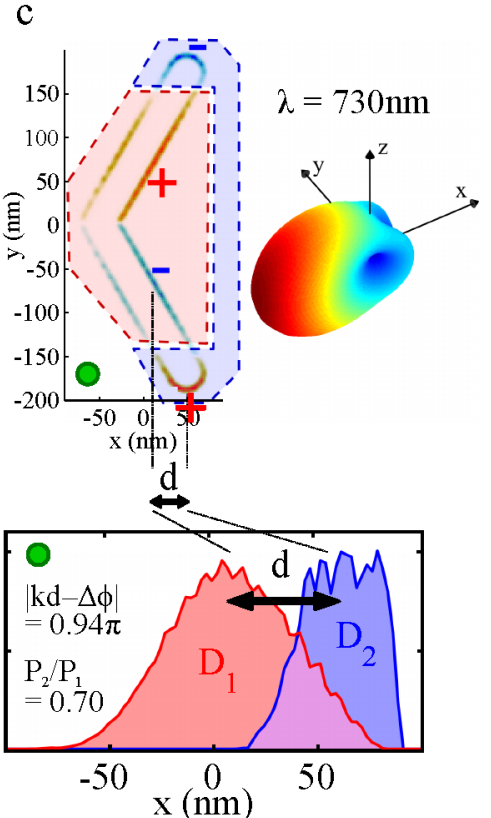

Figure 4. Two-dipole representation of the V-antenna $\mathrm{Q}_{4}$-Fano mode. (a) Illustration of directional emission (black line) from two point-dipole sources $\mathrm{D}_{1}$ (red) and $\mathrm{D}_{2}$ (blue) separated by a distance $d$. (b) Top: Simulated charge density distribution and 3D scattering distribution of a Vantenna $\left(L=250 \mathrm{~nm}, \alpha=120^{\circ}\right)$ in a homogeneous medium $(n=1)$ at $\lambda=650 \mathrm{~nm}$ (low directivity, orange dot). Bottom: Dipole moment $p_{y}$ as a function of the position $x$ along the antenna, calculated from the charge distribution. The dashed areas indicate the regions in which $\mathrm{D}_{1}$ and $\mathrm{D}_{2}$ are evaluated. $d$ indicates the distance between the center of $\mathrm{D}_{1}$ and $\mathrm{D}_{2}$. (c) Same as panel b, but $\lambda=730 \mathrm{~nm}$ (strongly directional scattering, green dot). (d) Spectral dependence of, from left to right: The antenna's extinction (black), absorption (green), and scattering (red) intensity at the $\mathrm{Q}_{y}$-Fano resonance; dipole moment strength, $P$, for $\mathrm{D}_{1}$ (red line) and $\mathrm{D}_{2}$ (blue line); phase difference $\Delta \phi-\pi$ of dipole moments $P_{1}$ and $P_{2}$, and phase shift $k d$ introduced by distance between $\mathrm{D}_{1}$ and $\mathrm{D}_{2}$; directivity derived from the two-dipole model.

the corresponding direction. Simultaneously, in the opposite direction the phase difference is lowered, resulting in constructive interference. Therefore, to obtain complete suppression of radiation in one direction, first, the dipole sources must have dipole moments of equal magnitude, and second, their phase difference must match the spatial separation such that $|k d \pm \Delta \phi|=\pi$.

The two-dipoles representation of the $\mathrm{V}$-antenna's $\mathrm{Q}_{y}$-Fano mode is based on the associated charge distributions. Figure 4 shows the charge distribution in a spectral region with low directivity (panel $b$, orange dot) and in one with high directivity (panel c, green dot), together with the corresponding 3D scattering plots. The antenna's spectral properties are shown in the left graph of panel $d$. In contrast to Figure 2, here, the substrate was not included in the simulation ( $\mathrm{V}$-antenna in vacuum, $n=1)$. Consequently, the forward/backward scattering symmetry along the $z$-axis is restored (panels b,c). From the charge distributions two regions, $\mathrm{D}_{1}$ and $\mathrm{D}_{2}$, can be defined for each wavelength. These regions are indicated with the dashed areas in Figure $4 b, c$ and represent the two dipole sources in the model. To determine the strength, phase, and position of $\mathrm{D}_{1}$ and $\mathrm{D}_{2}$, their respective dipole moments $p_{y}$ as a function of the position $x$ along the antenna are calculated:

$$
p_{y}^{i}(x, \lambda)=\iint_{\mathrm{D}_{i}(x, \lambda)} \rho(x, y, z, \lambda) y \mathrm{~d} y \mathrm{~d} z
$$

with $\rho$ the complex charge density and $i=1,2$ referring to the two dipoles. $p_{y}^{i}(x)$ for $\mathrm{D}_{1}$ (red curve) and $\mathrm{D}_{2}$ (blue curve) are shown at the bottom of panels $b$ and $c$. From these curves we obtain, first of all, the positions of $\mathrm{D}_{1}$ and $\mathrm{D}_{2}$ as the average position weighted by $p_{y}^{i}(x)$, and consequently their separation distance $d$. By calculating the total dipole moment, $P$, given by

$$
P_{i}(\lambda)=\int_{\mathrm{D}_{i}(\lambda)} p_{y}^{i}(x, \lambda) \mathrm{d} x
$$

for both dipoles, their relative strength and phase difference $\Delta \phi$ can be evaluated as well. The full spectral dependence of the dipole strength $P$ is shown in the second graph of Figure $4 \mathrm{~d}-$ $\mathrm{D}_{1}$ (red curve) and $\mathrm{D}_{2}$ (blue curve). The next graph depicts $\Delta \phi$ $-\pi$ and $k d$.

On the shorter wavelength side of the Fano resonance (orange dots), the increased scattering intensity can be attributed to the dominating $\mathrm{D}_{2}\left(P_{2} / P_{1}=1.88\right)$. Simultaneously, panel $\mathrm{b}$ shows charge accumulation on the outer edges of the antenna, resulting in a large separation with $k d=0.24 \pi$ rad. Since $\Delta \phi$ is only $1.03 \pi \mathrm{rad}$, it cannot compensate the large spatial phase difference $k d$. We get at best $|k d-\Delta \phi|=0.79 \pi$ $\mathrm{rad}$, and almost no directionality is obtained. On the longer wavelength side of the Fano resonance, very different behavior is observed (green dots). The destructive Fano interference translates into dipoles $\mathrm{D}_{1}$ and $\mathrm{D}_{2}$ with comparable moments $\left(P_{2} / P_{1}=0.70\right)$, as can be seen again in panel d. Furthermore, the charges that makes up $D_{1}$ and $D_{2}$ are now located near the center of the antenna, giving rise to a small separation $(k d=$ $0.14 \pi \mathrm{rad})$. Additionally, $\Delta \phi$ rises gradually with the wavelength, up to $\Delta \phi=1.08 \pi \mathrm{rad}$. The spatial phase shift now closely matches $\Delta \phi$ such that $|k d-\Delta \phi|=0.94 \pi$ rad. Consequently, the requirements for directional emission are met, and a highly unidirectional $3 \mathrm{D}$ scattering distribution is observed (panel c). For wavelengths above $\sim 750 \mathrm{~nm}$, not much of the Fano interference remains as we are far from the $Q_{y}$ mode. Only the $\mathrm{D}_{y}$ contribution remains, and as expected, one of the dipoles in the model, $\mathrm{D}_{2}$, disappears. 
Finally, also the directivity can be derived from the twodipole model:

$$
D(\lambda)=\left|10 \log _{10} \frac{\left|P_{1}+\mathrm{e}^{+i k d} P_{2}\right|^{2}}{\left|P_{1}+\mathrm{e}^{-i k d} P_{2}\right|^{2}}\right|
$$

$D(\lambda)$-plotted in Figure $4 \mathrm{~d}$, right graph-reproduces the spectral behavior of the directivity observed in the experiments and as calculated from the simulated far-field projections (Figure 2) very well. The maximum directivity is reached at $\lambda=$ $710 \mathrm{~nm}\left(P_{1} / P_{2}=0.95 \approx 1,|k d-\Delta \phi|=0.91 \pi \mathrm{rad}\right)$. This is very close to the amplitude matching point (intersection of red and blue curves-circle, panel d), yet shifted slightly toward the phase matching point (intersection of $k d$ and $\Delta \phi-\pi$ curvescircle) at $\lambda=770 \mathrm{~nm}$. Both the amplitude matching point and phase matching point are red-shifted relative to the extinction resonance. This is expected since both the amplitude and phase matching are conducive to a low net dipole moment which is provided by the destructive interference located at the longer wavelength side of the Fano resonance. The overall scattering cross-section of the antenna is therefore reduced at the maximum directivity. From the two point-dipoles model (panel a) it furthermore follows that varying the spectral distance between the amplitude and phase matching points results in a tunable maximum directivity. Since these points are ultimately determined by the exact distribution of the oscillating charges throughout the Fano resonance, and since these charges are bound by the antenna shape, it can be understood that changing the $\mathrm{V}$-angle of the antenna will affect the consequent achievable directivities, as was indeed observed in Figure 3.

To summarize, we have experimentally investigated the scattering properties of $\mathrm{V}$-shaped metallic nanoantennas. Unidirectional scattering of a plane wave in a direction perpendicular to the incident light direction was observed in FDTD simulations and back focal plane measurements, showing excellent mutual agreement. Tuning of the directivity was experimentally demonstrated by changing the $\mathrm{V}$-angle. An experimental left/right directional gain as large as $15 \mathrm{~dB}$ was achieved. In contrast to previous reports on directional side scattering, here the directionality phenomenon has been reduced to the single nanoparticle level. The antenna's reduced structural symmetry was shown to give rise to Fano interference of spectrally, and spatially, overlapping quadrupolar and dipolar LSPR antenna modes. Decomposing the antenna's charge oscillations in two dipole sources of variable amplitude, phase, and spatial separation, illustrated how this Fano interference can result in a tunable directivity that peaks in the resonance flank where the interference is destructive. Other asymmetric particle geometries supporting Fano resonances are expected to exhibit similar directional scattering properties, allowing further optimization. We believe that this concept can give rise to improvements in plasmon-based chemical and biological sensing, as well as surface enhanced spectroscopy. In addition, small $\left(<\lambda^{3} / 100\right)$ plasmonic directional antennas as these can form a promising base for compact directional emitters and constitute a single-element Yagi-Uda antenna.

\section{ASSOCIATED CONTENT}

\section{S Supporting Information}

More details on the BFP setup, BFP image processing, and calculation of the scattering distributions. Experimental spectral tunability data of the $\mathrm{V}$-antenna. Alternative directivity calculations. This material is available free of charge via the Internet at http://pubs.acs.org.

\section{AUTHOR INFORMATION}

\section{Corresponding Author}

*E-mail: Dries.Vercruysse@imec.be; Niels.Verellen@fys. kuleuven.be.

\section{Notes}

The authors declare no competing financial interest.

\section{ACKNOWLEDGMENTS}

D.V. acknowledges the I.W.T. Flanders, and N.V. acknowledges the F.W.O. (Flanders) for financial support. N.V. and V.V.M. are supported by the Methusalem funding by the Flemish Government. S.A.M and Y.S. acknowledge funding from the EPSRC, the Leverhulme trust, and the European Science Foundation. The authors thank Jos Moonens for e-beam assistance and Hector Sanchez for preliminary measurements with the BFP microscopy setup.

\section{REFERENCES}

(1) Loo, C.; Lowery, A.; Halas, N.; West, J.; Drezek, R. Nano Lett. 2005, 5, 709-711.

(2) Hu, M.; Chen, J.; Li, Z.-Y.; Au, L.; Hartland, G. V.; Li, X.; Marquez, M.; Xia, Y. Chem. Soc. Rev. 2006, 35, 1084.

(3) Mayer, K. M.; Hafner, J. H. Chem. Rev. 2011, 111, 3828-3857.

(4) Atwater, H. A.; Polman, A. Nat. Mater. 2010, 9, 205-213.

(5) Schuller, J. A.; Barnard, E. S.; Cai, W.; Jun, Y. C.; White, J. S.; Brongersma, M. L. Nat. Mater. 2010, 9, 193-204.

(6) Niesen, B.; Rand, B. P.; Van Dorpe, P.; Cheyns, D.; Tong, L.; Dmitriev, A.; Heremans, P. Adv. Energy Mater. 2012, 3, 145-150.

(7) Neutens, P.; Van Dorpe, P.; De Vlaminck, I.; Lagae, L.; Borghs, G. Nat. Photonics 2009, 3, 283-286.

(8) Lal, S.; Link, S.; Halas, N. J. Nat. Photonics 2007, 1, 641-648.

(9) Nordlander, P.; Oubre, C.; Prodan, E.; Li, K.; Stockman, M. I. Nano Lett. 2004, 4, 899-903.

(10) Verellen, N.; Sonnefraud, Y.; Sobhani, H.; Hao, F.; Moshchalkov, V. V.; Van Dorpe, P.; Nordlander, P.; Maier, S. A. Nano Lett. 2009, 9, 1663-1667.

(11) Luk'yanchuk, B.; Zheludev, N. I.; Maier, S. A.; Halas, N. J.; Nordlander, P.; Giessen, H.; Chong, C. T. Nat. Mater. 2010, 9, 707715 .

(12) Fan, J. A.; Wu, C.; Bao, K.; Bao, J.; Bardhan, R.; Halas, N. J.; Manoharan, V. N.; Peter, N.; Shvets, G.; Capasso, F. Science 2010, 328, 1135-1138.

(13) Sonnefraud, Y.; Koh, A. L.; McComb, D. W.; Maier, S. A. Laser Photonics Rev. 2012, 6, 277-295.

(14) Liu, W.; Miroshnichenko, A. E.; Neshev, D. N.; Kivshar, Y. S. ACS Nano 2012, 6, 5489-5497.

(15) Fu, Y. H.; Kuznetsov, A. I.; Miroshnichenko, A. E.; Yu, Y. F.; Lukyanchuk, B. Nat. Commun. 2013, 4, 1527.

(16) Luk'yanchuk, B. S.; Tribelsky, M. I.; Wang, Z. B.; Zhou, Y.; Hong, M. H.; Shi, L. P.; Chong, T. C. Appl. Phys. A: Mater. Sci. 2007, 89, 259-264.

(17) Mirin, N. A.; Halas, N. J. Nano Lett. 2009, 9, 1255-1259.

(18) Zhang, Y.; Barhoumi, A.; Lassiter, J. B.; Halas, N. J. Nano Lett. 2011, 11, 1838-1844.

(19) Van Dorpe, P.; Ye, J. ACS Nano 2011, 9, 6774-6778.

(20) Curto, A. G.; Volpe, G.; Taminiau, T. H.; Kreuzer, M. P.; Quidant, R.; van Hulst, N. F. Science 2010, 329, 930-933.

(21) Kosako, T.; Kadoya, Y.; Hofmann, H. F. Nat. Photonics 2010, 4, $312-315$.

(22) Maksymov, I. S.; Staude, I.; Miroshnichenko, A. E.; Kivshar, Y. S. Nanophotonics 2012, 1, 65-81.

(23) Dregely, D.; Taubert, R.; Dorfmüller, J.; Vogelgesang, R.; Kern, K.; Giessen, H. Nat. Commun. 2011, 2, 267. 
(24) Shegai, T.; Chen, S.; Miljković, V. D.; Zengin, G.; Johansson, P.; Käll, M. Nat. Commun. 2011, 2, 481.

(25) Shegai, T.; Johansson, P.; Langhammer, C.; Käll, M. Nano Lett. 2012, 12, 2464-2469.

(26) Pakizeh, T.; Käll, M. Nano Lett. 2009, 9, 2343-2349.

(27) Li, Z.; Hao, F.; Huang, Y.; Fang, Y.; Nordlander, P.; Xu, H. Nano Lett. 2009, 9, 4383-4386.

(28) Shegai, T.; Miljković, V. D.; Bao, K.; Xu, H.; Nordlander, P.; Johansson, P.; Käll, M. Nano Lett. 2011, 11, 706-711.

(29) Pellegrini, G.; Mazzoldi, P.; Mattei, G. J. Phys. Chem. C 2012 , 116, 21536-21546.

(30) Kats, M. A.; Genevet, P.; Aoust, G.; Yu, N.; Blanchard, R.; Aieta, F.; Gaburro, Z.; Capasso, F. Proc. Natl. Acad. Sci. U.S.A. 2012, 109, 12364-12368.

(31) Aieta, F.; Genevet, P.; Kats, M. A.; Yu, N.; Blanchard, R.; Gaburro, Z.; Capasso, F. Nano Lett. 2012, 12, 4932-4936.

(32) Sonnefraud, Y.; Kerman, S.; Di Martino, G.; Lei, D. Y.; Maier, S. A. Opt. Express 2012, 20, 4893-4902.

(33) Verellen, N.; Van Dorpe, P.; Huang, C.; Lodewijks, K.; Vandenbosch, G. A. E.; Lagae, L.; Moshchalkov, V. V. Nano Lett. 2011, 11, 391-397.

(34) Lumerical Solutions, Inc. http://www.lumerical.com.

(35) Johnson, P. B.; Christy, R. W. Phys. Rev. B 1972, 6, 4370.

(36) Bohren, C. F.; Huffman, D. R. Absorption and Scattering of Light by Small Particles; John Wiley \& Sons: New York, 1983.

(37) Khlebtsov, B. N.; Khlebtsov, N. G. J. Phys. Chem. C 2007, 111, $11516-11527$.

(38) Verellen, N.; Van Dorpe, P.; Vercruysse, D.; Vandenbosch, G. A. E.; Moshchalkov, V. V. Opt. Express 2011, 19, 11034-11051.

(39) López-Tejeira, F.; Paniagua-Domínguez, R.; Rodríguez-Oliveros, R.; Sánchez-Gil, J. A. New J. Phys. 2012, 14, 023035.

(40) Taminiau, T. H.; Stefani, F. D.; van Hulst, N. F. Nano Lett. 2011, 11, 1020-1024. 